\title{
Las melodías de los enunciados interrogativos con marca sintáctica del alemán septentrional en habla espontánea ${ }^{1}$
}

\author{
José Torregrosa Azor - Universidad de Barcelona \\ jtorregrosa@ub.edu
}

Rebut / Received: 23-9-15

Acceptat / Accepted: 22-12-15

Resum. Les melodies dels enunciats interrogatius amb marca sintàctica de l'alemany septentrional en parla espontània. L'anàlisi de l'entonació de l'alemany, així com també d'algunes de les seves varietats lingüístiques, ha experimentat un augment notable a partir de l'aplicació del mètode metricoautosegmental desenvolupat inicialment per Pierrehumbert (1980) i adaptat posteriorment a l'àmbit alemany per Grice et al. (1996), Gussenhoven (1988) i Mayer (1995). El procediment analític que s'aplica en aquest treball és metodològicament diferent al mètode metricoautosegmental. L'anàlisi acústica dels enunciats seleccionats es du a terme a través del mètode d'anàlisi melòdica de la parla (AMP), desenvolupat per Cantero (2002). El principal objectiu que ens proposem és analitzar, descriure i caracteritzar els patrons melòdics dels enunciats interrogatius amb marca sintàctica de l'alemany septentrional en parla espontània a través del mètode AMP i comparar els resultats obtinguts amb els que es descriuen a partir de l'aplicació de la metodologia mètrica i autosegmental.

Paraules clau: anàlisi melòdica de la parla (AMP), entonació de l'alemany, parla espontània, patrons melòdics, interrogatives absolutes.

\footnotetext{
Abstract. The melodies of Northern German syntactically marked interrogative utterances in spontaneous speech. Research on German intonation has been notably increasing since the metrical-autosegmental system was applied to the description of German intonation patterns

1. Este trabajo se inscribe en el proyecto de investigación Análisis melódico del habla y modelos didácticos, $\mathrm{n}^{\circ}$ de ref. FFI2013-41915-P, financiado por el Ministerio de Economía y Competitividad.
} 
and also to the intonation description of some German linguistic varieties. This system for the description of intonation was developed first by Pierrehumbert (1980) and later adapted to German by Grice et al. (1996), Gussenhoven (1988) and Mayer (1995). The analytical procedure, that we apply in the present work, is methodologically quite different from the metrical-autosegmental framework. The acoustic analysis of utterances will be carried out with the melodic analysis of speech (MAS) method, developed by Cantero (2002). The objective of this paper is to analyse, to describe and to characterise the melodic patterns of syntactically marked questions in northern German in spontaneous speech and to compare our results with the descriptions of German intonation patterns obtained through the metrical-autosegmental system.

Key words: Melodic Analysis of Speech (MAS), German intonation, spontaneous speech, melodic patterns, yes/no questions.

\section{Introducción}

El estudio de la entonación ha experimentado un gran desarrollo disciplinar a partir de las últimas décadas del sigo XX, que en gran medida ha sido propiciado por la creación de potentes instrumentos de análisis del material acústico. Los avances tecnológicos y la aportación metodológica de diferentes perspectivas teóricas han dado lugar a una gran producción científica que ha contribuido a una mejor comprensión de la prosodia de las lenguas.

En este sentido, merecen especial mención los estudios comparativos, tanto desde un punto de vista interlingüístico comparando las características prosódicas afines y diferentes entre lenguas, pertenecientes o no a una misma familia lingüística, como también desde un punto de vista intralingüístico en el que destacan los trabajos comparativos entre las diferentes variedades lingüísticas de una lengua dada.

Este es el caso de los trabajos que describen las características prosódicas de las lenguas y sus variantes lingüísticas, tanto del ámbito lingüístico románico (Cantero 1999, Cantero 2002, Font-Rotchés 2007, Rossano 2010, Martínez Celdrán y Fernández Planas 20032012, Dorta 2013, Frota y Prieto 2015 y Aronsson 2015), como en el ámbito lingüístico germánico (Ladd 1996, Grabe 1998, Grabe et al. 2005, Gussenhoven 2005, Fletcher et al. 2006, Peters 2006, Kügler 2007, Ramírez Verdugo et al. 2007 y Leeman 2012).

Considerando los estudios realizados en este último grupo lingüístico, el análisis de la entonación se ha realizado principalmente a través de la perspectiva métricaautosegmental. En el presente trabajo, que se enmarca dentro del análisis de la variación prosódica intralingüística del alemán, presentamos una perspectiva metodológica distinta, el análisis melódico del habla (AMH), a través del cual describimos las 
características melódicas de los enunciados interrogativos con marca sintáctica del alemán septentrional en habla espontánea.

\section{La entonación de las interrogativas con marca sintáctica en alemán}

La entonación de las interrogativas absolutas del alemán ha sido analizada y descrita a partir de diversas metodologías, pero sobre todo a través del sistema métricoautosegmental desarrollado inicialmente por Pierrehumbert (1980), más conocido como sistema Tones and Breaks Indices (ToBI). En el contexto alemán, este modelo de análisis de la entonación ha desarrollado su propio sistema conformando el conjunto de convenciones para la descripción de la entonación del alemán o German Tones and Break Indices (GToBI) (Grice et al. 1996).

El sistema ToBI, así como GToBI describen la entonación a partir de la expresión melódica del último acento tónico del enunciado, el núcleo tonal o pitch accent. La expresión melódica del núcleo se define en función de dos niveles tonales, un nivel alto $(\mathrm{H})$ o un nivel bajo $(\mathrm{L})$, pudiendo realizarse de forma monotonal o también de forma bitonal, la cual resultaría de la combinación de los dos niveles tonales indicados anteriormente. Para describir la trayectoria de la IF, que se desarrolla a partir del núcleo tonal del enunciado, el sistema métrico-autosegmental considera una segunda categoría de niveles tonales, llamados tonos de frontera o boundary tones, que se asocia con el final del enunciado y se etiquetan como alto o bajo dependiendo de la trayectoria que desarrolla la IF, pero incluyendo el diacrítico (\%) a la derecha del tono, $(\mathrm{H} \%)$ o bien (L\%).

Concretamente, a través de la aplicación del modelo GToBI (Baumann et al. 2001 y Grice et al. 2005) se exponen 4 patrones entonativos diferentes para las interrogativas absolutas del alto alemán estándar.

Tres de estos cuatro patrones descritos presentan una terminación ascendente con variaciones centradas en la expresión del núcleo, a partir del cual se desarrolla la IF: a) $\mathrm{L}^{*} \mathrm{H}-{ }^{\wedge} \mathrm{H} \%$ o, según Kügler (2004), L*H; b) L*L-H\%, y c) (L+) $\mathrm{H}^{*} \mathrm{H}-{ }^{\wedge} \mathrm{H} \%$. El cuarto patrón también presenta un final ascendente, pero precedido por un descenso que no se observa en los otros tres patrones: d) $\mathrm{H}^{*} \mathrm{~L}-\mathrm{H} \%$.

Uhmann (1991) describe solo dos patrones para las interrogativas absolutas y en ambos casos el final es ascendente: $\mathrm{L}^{*}+\mathrm{HH} \%$ y $\mathrm{H}^{*}+\mathrm{LH} \%$.

En Kügler (2004) se relacionan los mismos patrones entonativos descritos en Uhmann (1991), pero incluye otros dos patrones con final descendente (H*LL\% y L*HL\%) para las interrogativas absolutas, aunque observa diferencias de uso dependiendo de la variedad lingüística. Mientras que los cuatro patrones entonativos $\mathrm{L}^{*} \mathrm{HH} \%, \mathrm{H}^{*} \mathrm{LH} \%$, $\mathrm{H}^{*} \mathrm{LL} \%$ y L*HL\% los describe dentro del suabo, una variedad lingüística meridional de Alemania, solo L*HH\% y L*HL\% los describe en alto sajón, una variedad lingüística en la zona geográfica de Leipzig. 
Peters (2005) describe 4 patrones diferentes para las interrogativas absolutas: $\mathrm{H}^{*} \mathrm{~L} \rightarrow \mathrm{L} \% ; \mathrm{H}^{*} \mathrm{~L} \rightarrow \mathrm{H} \% ; \mathrm{H}^{*} \mathrm{LH} \%$ y $\mathrm{H}^{*} \mathrm{LL} \%$.

En síntesis, observamos discrepancias en relación con la descripción de la entonación de los enunciados interrogativos absolutos del alemán. Mientras que para Uhmann (1991) se realizan solo con final ascendente, para la mayoría de autores pueden contemplar tanto final ascendente como descendente.

También observamos que a medida que avanzan los estudios sobre la entonación del alemán y que se realizan más análisis de enunciados, también se observan novedades en la configuración melódica, que se traducen con la adición de nuevos elementos diacríticos en el sistema de etiquetado y que redundan en un desarrollo complejo del sistema de notación.

Finalmente, otro aspecto que hemos observado de forma general es un protocolo de grabaciones en condiciones de laboratorio que minimiza el factor espontaneidad y un uso limitado de informantes (Grabe 1998, Ambrazaitis 2008 y Petrone y Niebuhr 2014), de los cuales generalmente no se indica distinción de género (Kügler 2004) o, si se indica, suelen corresponder al mismo sexo (Grabe 1998 y Ambrazaitis 2008) o si los informantes son de distinto género (Kügler 2004 y Petrone y Niebuhr 2014) no se indica qué tipo de correlación se sigue para gestionar la diferencia de rango tonal que diferencia la voz femenina de la masculina y poder comparar los contornos melódicos resultantes.

\section{Materiales y método de análisis}

\subsection{Corpus de datos}

El corpus de datos seleccionado consta de 85 enunciados interrogativos con marca sintáctica emitidos por 22 informantes, 14 mujeres y 8 hombres, de edades comprendidas entre 17 y 38 años. Las grabaciones audiovisuales proceden de diversos canales y programas de la televisión alemana, así como también entrevistas grabadas en la calle y en diversas localidades del norte de Alemania (Berlín, Hamburgo y Münster).

Los programas de televisión corresponden a los géneros talk show y live talk. Esta modalidad de programas, así como también las entrevistas realizadas en la calle, son especialmente aptas para un tipo de investigación en el que se analice la entonación a partir de un registro lingüístico natural y cotidiano, como el que se propone en este trabajo, porque ofrecen la ventaja de poder obtener emisiones orales genuinas de un número de informantes muy amplio y heterogéneo, tanto en las variables de edad como de sexo, de formación, de profesión, etc., que garantiza la obtención de un corpus oral y espontáneo de la lengua objeto de estudio.

El análisis acústico de los datos se realiza mediante el programa Praat (Boersma y Weenink 1992-2012) y la posterior interpretación melódica a través del método AMH que se describe a continuación. 


\subsection{Método de análisis}

En esta sección presentamos el método AMH (Cantero 2002) que permite obtener una representación melódica generalizable de los enunciados teniendo en cuenta la información fónica relevante contenida en los mismos y discriminando aquella que no lo es. Este método de análisis está basado en el principio de jerarquía fónica, según el cual los sonidos del habla no son una mera sucesión de emisiones aisladas, sino que se organizan en bloques fónicos, el núcleo de los cuales es un segmento vocálico (Cantero 2002, p. 147).

Desde esta perspectiva, los elementos de la cadena fónica se encuentran perfectamente estructurados y jerarquizados alrededor de los sonidos vocálicos, que son los elementos que aportan información melódica relevante cumpliendo una función integradora dentro de la estructura discursiva. Por tanto, la unidad de análisis es el segmento vocálico, ya que es el elemento de la cadena fónica que tiene tono y del cual se puede obtener la frecuencia fundamental (F0). Así, el procedimiento que se sigue consiste en obtener y anotar los valores frecuenciales absolutos $(\mathrm{Hz})$ de cada uno de los sonidos vocálicos de que se compone el enunciado.

En la Figura 1 se muestra un ejemplo de extracción de la F0. Las zonas enmarcadas indican los diferentes puntos de extracción de la F0 de los diferentes segmentos vocálicos.

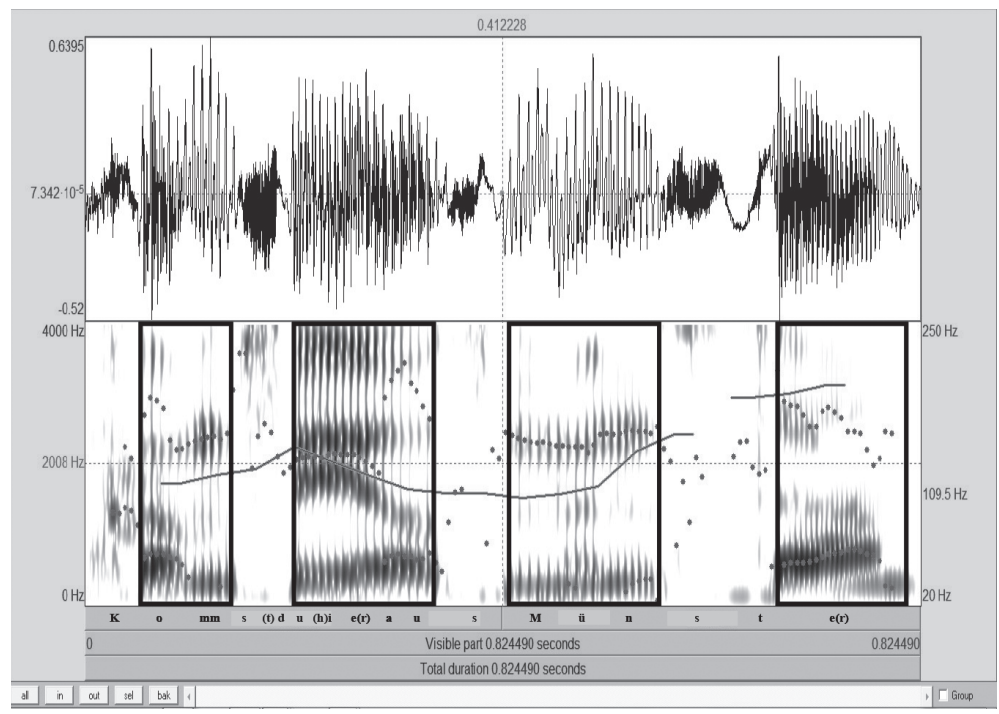

Figura 1. Extracción de los valores frecuenciales del enunciado Kommst $d u$ hier aus Münster? ('¿Vienes de aquí de Münster?’). 
Como se puede observar, las zonas enmarcadas se corresponden con las bandas oscuras del espectrograma en las que se localizan los segmentos vocálicos que componen el enunciado. De cada uno de estos segmentos se obtiene el valor de la frecuencia fundamental (F0) en Hz. Estos valores se trasladan posteriormente a una tabla de anotación que hemos preparado previamente (Tabla 1).

Generalmente, se obtiene un solo valor frecuencial por cada segmento silábico que compone el enunciado. Sin embargo, en ocasiones se pueden observar inflexiones tonales que pueden afectar a un mismo segmento. En tal caso, cuando en la inflexión tonal se observa un contraste tonal superior al $10 \%$, sea de aumento o de disminución, se anotan los valores tonales correspondientes, que en la mayoría de los casos suelen ser dos, aunque en ocasiones se pueden llegar a registrar hasta tres valores tonales cuando la inflexión tonal que se produce es circunfleja -ascendente/descendente o descendente/ascendente.

Tabla i. Tabla de anotación de los valores Fo en Hz.

\begin{tabular}{|c|c|c|c|c|c|c|}
\hline Segmento & Kommst & du (h)ier aus & Müns & Müns* & ter & ter* \\
\hline HZ & 122 & 127 & 106 & 146 & 191 & 230 \\
\hline
\end{tabular}

En la Tabla 1 se observa el fenómeno referido anteriormente y afecta a las dos últimas sílabas del enunciado, Müns-y-ter. De cada una se han obtenido dos valores frecuenciales, el primero se determina a partir de los primeros pulsos glotales que se muestran en el espectrograma y el segundo, que se identifica con el símbolo “*”, se extrae a partir de los últimos pulsos glotales.

Por otra parte, en habla espontánea es frecuente observar elisiones o asimilaciones de determinados elementos de la cadena hablada y tales fenómenos pueden constatarse mediante el correspondiente análisis espectrográfico (Grabe 1998). En nuestro corpus de datos hemos optado por indicar entre paréntesis este tipo de fenómenos y seńalar de esta forma que se trata de un elemento elidido o asimilado y que no tiene una correspondencia acústica en el análisis espectrográfico. Este fenómeno también lo observamos en el enunciado representado en la Tabla 1, en el segmento $d u$ (h)ier aus. 


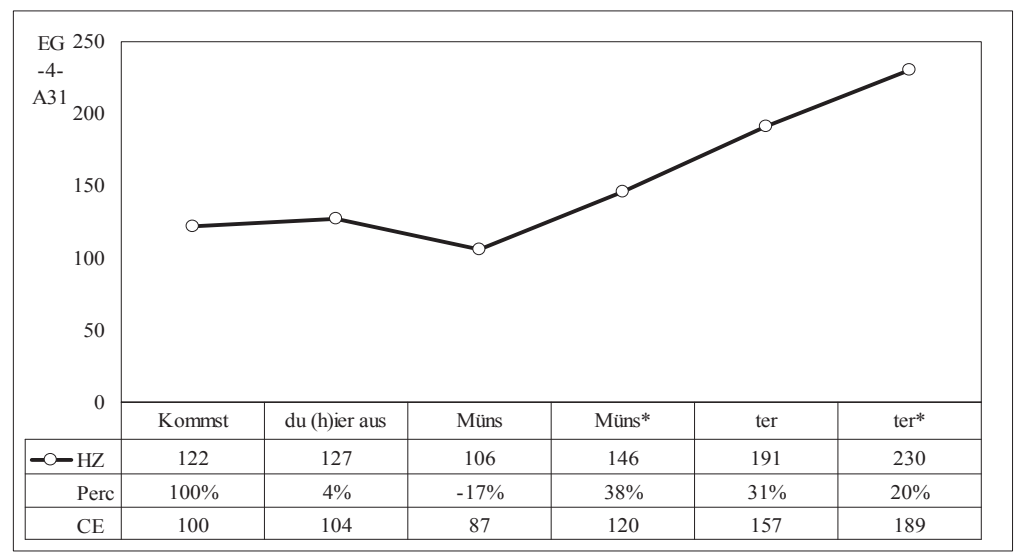

Figura 2. Contorno estilizado de los valores frecuenciales (F0) del enunciado Kommst du hier aus Münster? (‘¿Vienes de aquí de Münster?’).

El gráfico que resulta después de procesar los datos se genera a partir de las variaciones frecuenciales de los segmentos vocálicos y muestra la abstracción o estilización de la melodía del enunciado analizado.

El contorno que representamos en la Figura 2 corresponde a la curva estilizada obtenida a partir de los datos F0 recogidos de cada uno de los segmentos de que se compone.

Sin embargo, el contorno que se representa conserva todavía los rasgos fónicos individuales de cada informante, de cuyo análisis no es posible aún extraer generalizaciones comparables.

Es necesario, por tanto, obtener configuraciones que describan únicamente la estructura melódica de los enunciados prescindiendo de las características fónicas individuales para que los contornos resultantes puedan ser comparables con otras copias melódicas producidas por cualquier otro informante, independientemente de las variables de sexo o edad, y poder extraer y estudiar los rasgos melódicos comunes en una determinada variedad lingüística.

Por este motivo, es necesario llevar a cabo un proceso de relativización de estos datos, que consiste en transformar los valores frecuenciales en datos porcentuales que reflejan la relación tonal existente entre un valor absoluto y el valor absoluto inmediatamente posterior. De esta forma, la atención se focaliza en el diferencial tonal de los valores frecuenciales y no en los valores frecuenciales, prescindiendo de las variables micromelódicas intrínsecas a los rasgos fónicos de cada informante. 
Finalmente, se realiza un proceso de normalización, a través del cual se estandarizan los valores tomando como dato de referencia inicial el número 100, al cual se aplican los porcentajes obtenidos en el proceso de relativización. A continuación, presentamos las fórmulas de relativización y de estandarización, a partir de las cuales se genera la curva melódica estándar.

Fórmula de relativización ${ }^{2}: \quad$ VRELn+1 $=[($ VABSn $+1-V A B S n) / V A B S n] * 100$

Fórmula de estandarización: $\quad \mathrm{VSn}+1=\mathrm{VSn}+[(\mathrm{VRELn}+1 * \mathrm{VSn}) / 100]$

En la Tabla 2 se presenta la tabla de anotación completa después de que los valores frecuenciales (F0) se han procesado mediante la aplicación de las fórmulas de relativización y de estandarización. En la fila encabezada con "\%" se presentan los datos relativos obtenidos de la F0 que muestran la distancia tonal relativa existente entre los segmentos. Después de aplicar la fórmula descrita, en la fila encabezada con "CE" se presentan los valores estandarizados, a partir de los cuales se genera la configuración melódica estándar.

TABLA 2. TABLA DE ANOTACIÓN COMPLETA

\begin{tabular}{|c|c|c|c|c|c|c|}
\hline Segmento & Kommst & du (h)ier aus & Müns & Müns* & ter & ter* \\
\hline $\mathbf{H Z}$ & 122 & 127 & 106 & 146 & 191 & 230 \\
\hline$\%$ & $100 \%$ & $4 \%$ & $-17 \%$ & $38 \%$ & $31 \%$ & $20 \%$ \\
\hline $\mathbf{C E}$ & 100 & 104 & 87 & 120 & 157 & 189 \\
\hline
\end{tabular}

Aplicando el procedimiento descrito, se genera la curva melódica estándar, objetiva, comparable y generalizable desde un punto de vista lingüístico. La configuración resultante no se corresponde exactamente con la curva melódica pronunciada por el emisor, que ha sido generada a partir de los datos frecuenciales (F0), pero sí que es semejante, ya que de esta forma se centra la atención en los aspectos estrictamente tonales y posibilita que pueda ser comparada tonalmente con otras copias estandarizadas independientemente de la tesitura de los informantes que las han emitido. En el gráfico de la Figura 3 se representa el contorno estandarizado del enunciado Kommst du hier

2. VABSn corresponde al valor absoluto del segmento vocálico de referencia, mientras que VABSn+1 corresponde al valor frecuencial del segmento vocálico que sucede al valor absoluto de referencia. VRELn+1 identifica el diferencial porcentual entre el valor absoluto $\mathrm{VABSn}_{n+1}$ y el inmediatamente anterior VABSn. Finalmente, Vsn+1 corresponde al valor estandarizado de VRELn+1 con respecto a VSn. Este último es el valor estándar que actúa como valor de referencia. 
aus Münster? ('¿Vienes de aquí de Münster?'), que se ha obtenido a partir de los valores relativos (\%) de cada uno de los segmentos analizados.

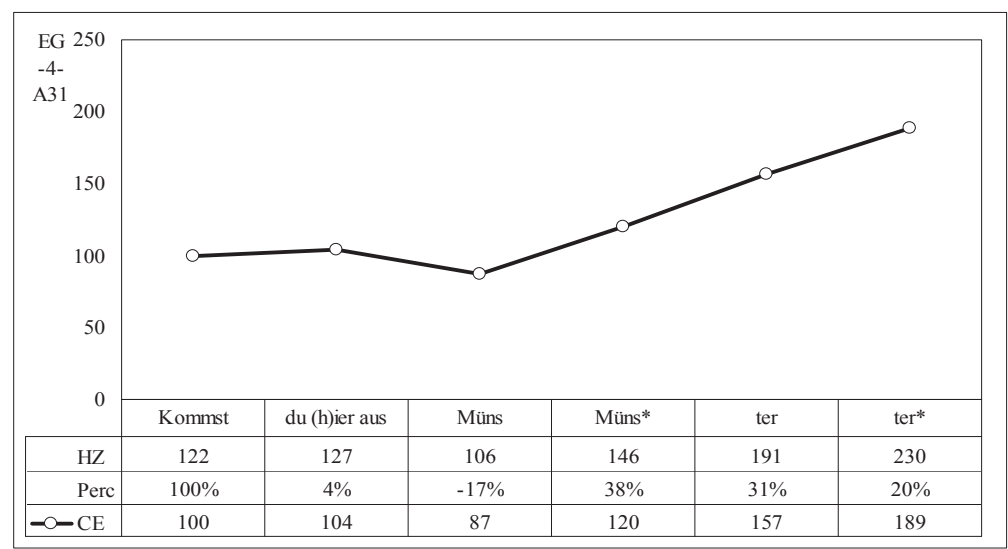

Figura 3. Representación estandarizada (CE) del enunciado Kommst du hier aus Münster? (‘¿Vienes de aquí de Münster?’).

Una vez se han obtenido todos los contornos estandarizados es necesario compararlos para establecer los aspectos fónicos que los asemejan y los que los diferencian. Para poder realizar la comparación entre contornos y la caracterización fonética y fonológica es necesario atender a las diferentes partes que lo componen y no únicamente a la inflexión final (IF), aunque ésta sea la que aporte la información lingüística más relevante: la anacrusis, el primer pico (1r P), el cuerpo del contorno, el núcleo $(\mathrm{N})$ y la IF.

En síntesis, en relación con otros modelos de análisis de la entonación -por ejemplo ToBI o AMPER-, el método AMH permite estudiar la entonación exclusivamente desde un punto de vista fonético y fonológico, sin que sea necesario recurrir a otros niveles de análisis - gramatical o pragmático, por ejemplo-. A su vez, permite trabajar con cualquier tipo de corpus -condicionado, semiespontáneo, habla espontánea genuina-, con un gran volumen de datos acústicos y con un gran número y diversidad de informantes.

\section{Resultados}

Después de haber procesado los 85 enunciados interrogativos con marca sintáctica (VSO) a través del método AMH e instrumentos descritos anteriormente, hemos obtenido 3 patrones melódicos distintos para los enunciados interrogativos con marca 
sintáctica del alemán septentrional en habla espontánea. Estos 3 patrones melódicos se describen a continuación.

\subsection{Patrón melódico IF descendente}

Desde un punto de vista melódico, el patrón representado en la Figura 4 se caracteriza por una IF con descenso tonal que generalmente no supera el 30\% hasta el último segmento del contorno. Se trata de enunciados que se formulan como preguntas en el contexto interaccional, cuya respuesta es sí o no.

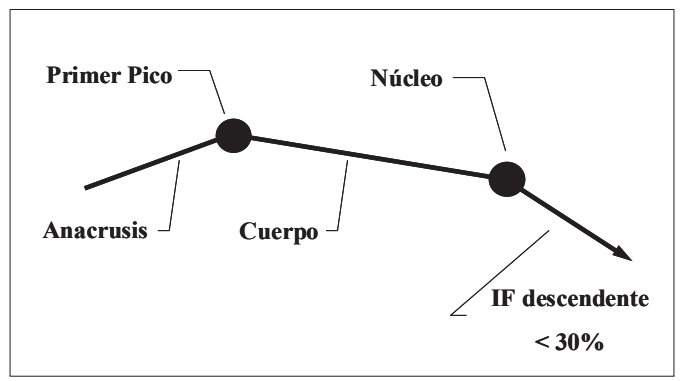

Figura 4. Patrón melódico con IF descendente.

Desde un punto de vista comunicativo, teniendo en cuenta la taxonomía que se ofrece en Escandell-Vidal (2009), la funcionalidad de este patrón melódico en contextos interactivos de habla espontánea es la de obtener información o también la de solicitar confirmación sobre la información dada previamente por el interlocutor.

La cantidad de enunciados en nuestro corpus de datos que se ajustan a las características descritas para este patrón melódico son en total 8 enunciados, que suponen el 9\% sobre el conjunto total.

Además de los rasgos melódicos en la IF, este contorno presenta otros dos rasgos melódicos más, uno en el primer pico y otro en el cuerpo del contorno. Con relación al primer pico, los contornos de este grupo presentan anacrusis opcional que, en el caso de que se produzca, muestra generalmente un ascenso tonal inferior al 30\% hasta el primer pico, coincidiendo con la primera sílaba tónica del contorno o desplazado en una átona posterior. Finalmente, el cuerpo del contorno suele presentar un descenso suave hasta la emisión del núcleo. En el gráfico de la Figura 5 se muestra un ejemplo de contorno melódico compatible con las características del patrón melódico descrito anteriormente. 


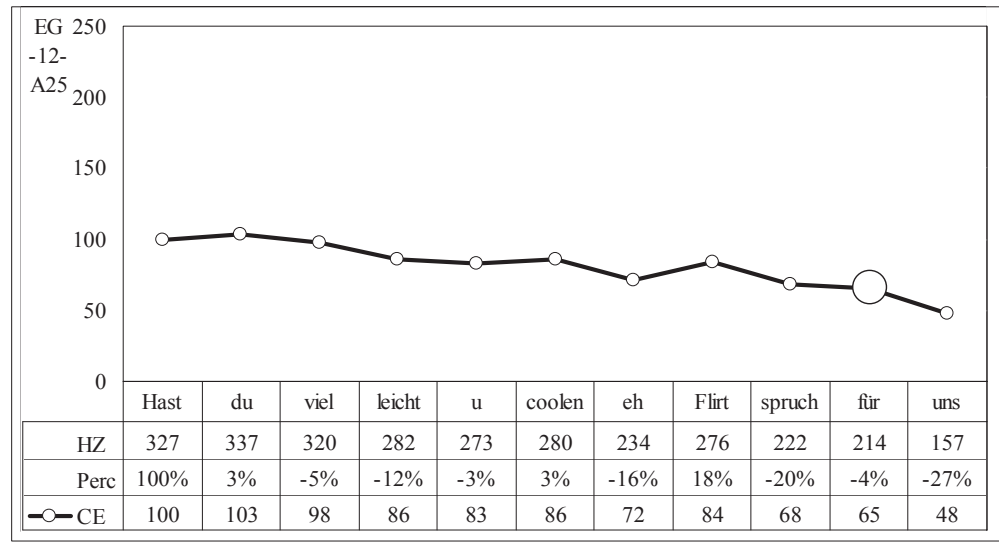

Figura 5. Contorno con IF descendente.

\subsection{Patrón melódico IF ascendente}

El patrón melódico que describimos a continuación y que mostramos en la Figura 6 presenta unos rasgos melódicos distintivos. Se caracteriza por una IF ascendente de hasta el 120\%, excepto algunos contornos que pueden alcanzar el 190\%.

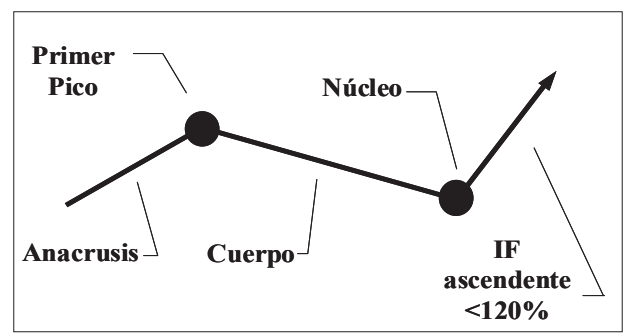

Figura 6. Patrón melódico con IF ascendente.

Se trata de enunciados que se formulan como preguntas en el contexto interaccional, cuya respuesta es sí o no. Los hablantes suelen utilizar este tipo de patrón melódico para obtener información, según la taxonomía que se propone en Escandell-Vidal (2009). En el caso de que el hablante necesite enfatizar el carácter interrogativo del enunciado, el 
rango de ascenso tonal que se desarrolla en la IF suele superar el 120\%. En total hemos obtenido 57 enunciados que se ajustan a las características descritas para este patrón melódico y suponen el $67 \%$ sobre el conjunto total.

Además de los rasgos melódicos en la IF, presenta otros dos rasgos melódicos más, uno en el primer pico y otro en el cuerpo del contorno. En relación con el primer pico, los contornos de este grupo presentan anacrusis opcional que, en el caso de que se produzca, muestra generalmente un ascenso tonal que no supera el $30 \%$ hasta la emisión del primer pico, que suele coincidir con la primera sílaba tónica o encontrarse desplazado en el segmento átono posterior. A partir del primer pico, se desarrolla el cuerpo con un descenso suave hasta la emisión del último segmento tónico del contorno que coincide con la emisión del núcleo, a partir del cual se desarrolla la IF.

En el contorno representado en la Figura 7 se muestra un ejemplo representativo que se ajusta a las características de este patrón melódico.

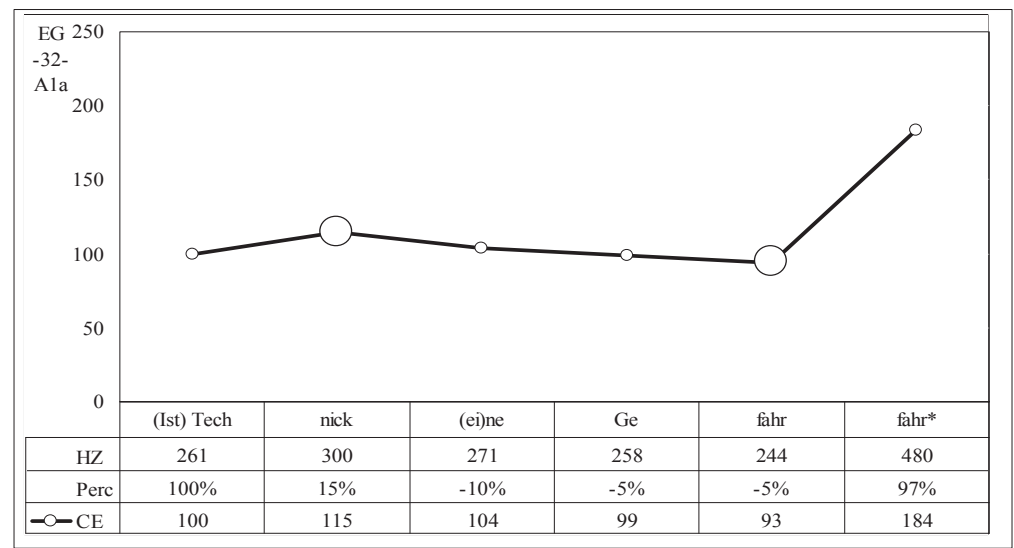

Figura 7. Contorno con IF ascendente.

\subsection{Patrón melódico con cuerpo e IF ascendentes}

El último patrón que presentamos y que se muestra en la Figura 8 se caracteriza melódicamente por describir en total un ascenso tonal variable de entre $40 \%$ y $140 \%$. Otro rasgo melódico característico es la ausencia de primer pico. 


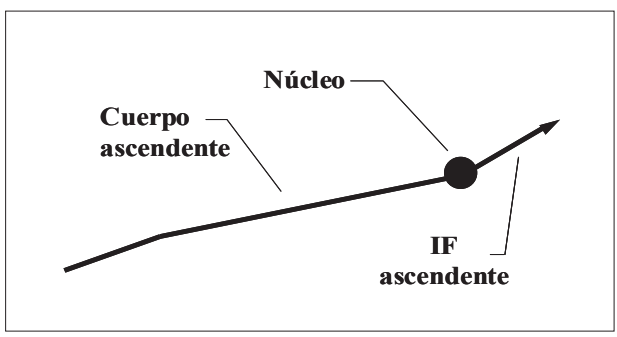

Figura 8. Patrón melódico con cuerpo e IF ascendentes.

La cantidad de enunciados en nuestro corpus que se ajustan a las características descritas para este patrón melódico es de 20 enunciados y suponen el 24\% sobre el conjunto total. En el gráfico de la Figura 9 mostramos un ejemplo de este patrón melódico.

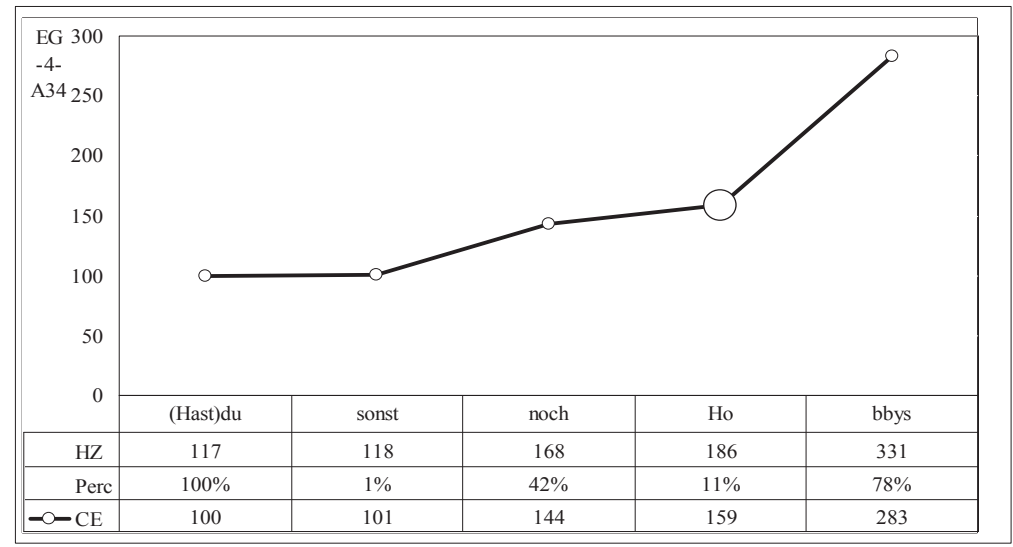

Figura 9. Contorno con cuerpo e IF ascendentes.

\section{Conclusión y futuras investigaciones}

El objetivo que nos proponíamos en este trabajo consistía, en primer lugar, en describir los patrones melódicos de enunciados interrogativos con marca sintáctica del 
alemán septentrional en habla espontánea a través del método AMH y, en segundo lugar, comparar los resultados obtenidos con las descripciones entonativas obtenidas a partir de la aplicación del sistema métrico-autosegmental.

A partir de nuestro corpus de datos de habla espontánea y a partir de la aplicación del modelo de análisis de la entonación AMH hemos establecido 3 patrones melódicos distintos que mencionamos seguidamente:

- Patrón melódico IF descendente.

- Patrón melódico IF ascendente.

- Patrón melódico con cuerpo e IF ascendentes.

Los dos primeros se describen a partir de la dirección y el porcentaje de movimiento tonal que presenta la línea melódica en la IF, mientras que el tercero se caracteriza por presentar un cuerpo ascendente seguido de IF ascendente. Cabe decir que no todos tienen el mismo rendimiento, ya que destaca el patrón melódico con IF ascendente, con un $67 \%$ de los contornos, seguido del patrón con cuerpo e IF ascendentes, con una presencia en nuestro corpus del $24 \%$ y, finalmente, del patrón con IF descendente, con un $9 \%$ de representatividad.

Comparando los resultados que hemos obtenido tras la aplicación del método AMH con las descripciones entonativas realizadas a través del sistema métrico-autosegmental, encontramos más divergencias que concurrencias.

Hemos obtenido consenso con relación al patrón melódico con IF ascendente, que podría ser equiparable a las siguientes descripciones: $\mathrm{L}^{*} \mathrm{H}-{ }^{\wedge} \mathrm{H} \%$ (Baumann et al. 2001 y Grice et al. 2005), L*H y L*HH\% (Kügler 2004) y L*HH\% y L+H* $\rightarrow \mathrm{H} \%$ (Peters 2005). En general, los autores que aplican el sistema métrico-autosegmental coinciden en señalar un desarrollo tonal ascendente a partir de la emisión del núcleo, pero no cuantifican el rango de aplicación de este ascenso tonal para los patrones que describen. A partir de los resultados que hemos obtenido coincidimos en la descripción de un patrón con IF ascendente que se desarrolla a partir de la emisión del núcleo. Sin embargo, nosotros sí cuantificamos con precisión el rango de aplicación del ascenso tonal que se desarrolla en la IF.

Por otra parte, las diferencias que se observan en las descripciones de los patrones descritos a través del sistema métrico-autosegmental pueden deberse, en primer lugar, por tomar en consideración únicamente las variaciones de F0 y, en segundo lugar, por el tipo de corpus utilizado. A diferencia del sistema métrico-autosegmental, que ofrece una amplia variedad de posibilidades para describir el ascenso tonal a partir de la emisión del núcleo, el patrón que describimos a través del método AMH es concreto, unívoco y con capacidad para representar cualquiera de las variantes melódicas ascendentes, también las descritas a través del sistema métrico-autosegmental, que concurran con las características descritas para el patrón con IF ascendente, ya que a través del método $\mathrm{AMH}$ sí es posible cuantificar con precisión el rango de actuación del patrón melódico. 
Otra característica importante que diferencia el patrón con IF ascendente obtenido a través de la aplicación del método AMH de los obtenidos a través del sistema métricoautosegmental consiste en que este patrón melódico es el resultado de comparar múltiples configuraciones de producciones orales espontáneas de múltiples informantes de distinto sexo. Como ya explicamos en la sección 3.2., a diferencia de otros sistemas de análisis de la entonación, a través del método AMH es posible comparar perfiles melódicos obtenidos a partir de diferentes informantes de distinto sexo o edad y obtener un patrón estándar que los represente, que los describa y que los defina melódicamente con precisión.

En relación con el patrón con IF descendente, solo podemos relacionarlo con las descripciones que se realizan en Kügler (2004) y en Peters (2005), ya que los patrones descritos en Baumann et al. (2001) y Grice et al. (2005) contemplan únicamente variaciones melódicas con IF ascendente para los enunciados, aunque también describen otros patrones con IF descendente, pero para enunciados interrogativos pronominales o con marca léxica.

En relación con el patrón con cuerpo e IF ascendente no hemos encontrado ninguna coincidencia con las descripciones que se realizan a través del sistema métricoautosegmental, ya que las descripciones que se exponen contemplan el desarrollo de la IF, pero generalmente no incluyen los movimientos tonales que se registran en el cuerpo y en la anacrusis del contorno melódico. Por otra parte, resulta notorio destacar que en nuestro corpus de datos, un corpus de datos obtenido a partir de producciones espontáneas en contextos interactivos reales y cotidianos, este patrón detenta una frecuencia de aparición del 24\%, a diferencia del patrón con IF descendente que refleja una frecuencia de uso del $9 \%$.

Finalmente, no hemos obtenido configuraciones que tracen otros movimientos tonales comparables con otras descripciones realizadas a través del sistema métricoautosegmental. Estos movimientos tonales se refieren a IF circunflejas, tanto ascendentedescendente ( $\mathrm{L}^{*} \mathrm{HL} \%$ ), descrita en Kügler (2004) para una variante lingüística meridional y otra septentrional, como descendente-ascendente ( $\left.\mathrm{H}^{*} \mathrm{LH} \%\right)$, descrita en Baumann et al. (2001) y Grice et al. (2005), también en Kügler (2004) para una variante lingüística meridional de Alemania y, finalmente en Peters (2005), que además del patrón circunflejo $\mathrm{H}^{*} \mathrm{LH} \%$ presenta otra variante melódica, $\mathrm{H}^{*} \mathrm{~L} \rightarrow \mathrm{H} \%$.

En conclusión, el trabajo que presentamos se trata de una aportación empírica inédita. Hasta el momento no se había caracterizado con tanta precisión y desde una metodología que aborda el análisis de la entonación desde un punto de vista estrictamente fonético tres patrones con rasgos tan distintos y valores exactos expresados en porcentajes de ascenso y de descenso, y obtenidos a partir de producciones espontáneas de hablantes nativos de alemán.

Sin embargo, a partir del nivel de descripción lingüístico de la entonación en que hemos centrado nuestra investigación, no es posible determinar la caracterización fonológica de 
los patrones entonativos o tonemas. Por este motivo, como futuras investigaciones nos hemos propuesto llevar a cabo pruebas perceptivas con informantes nativos de la lengua objeto de estudio con la finalidad de culminar el análisis de la entonación en el nivel de descripción lingüístico y determinar los tonemas de la modalidad interrogativa con marca sintáctica del alemán septentrional en habla espontánea.

\section{Referencias}

Ambrazaitis, G. (2008). On final rises and fall-rises in German and Swedish. En A. Eriksson y J. Lindh (eds.), Proceedings FONETIK 2008, the XXIst Swedish Phonetics Conference (p. 81-84). Gothenburg: University of Gothenburg. Disponible en: http://www.ling.gu.se/konferenser/fonetik2008/papers/Proc_fonetik_2008.pdf. Acceso: 01.10.2014.

Aronsson, B. (2015). Efectos pragmáticos de transferencias prosódicas del sueco al español L2: implicaciones para la clase de español lengua extranjera (Tesis doctoral). Umeå University, Suecia. Disponible en: http://www.diva-portal.org/smash/record. jsf?pid=diva2:849201. Acceso: 06.12.2015.

Baumann, S., Grice, M., y Benzmüller, R. (2001). GToBI -a phonological system for the transcription of German intonation. En S. Puppel y G. Demenko (eds.), Prosody 2000: speech recognition and synthesis (p. 21-28). Adam Mickiewicz University, Poznan.

Boersma, P., y Weenink, D. (1992-2012). PRAAT. Doing phonetics by computer. Ámsterdam: University of Amsterdam. Disponible en: http://www.praat.org. Acceso: 09.07.2008.

Cantero, F. J. (1999). Análisis melódico del habla: Principios teóricos y procedimiento. Actas del I Congreso de Fonética Experimental (p. 127-133). Tarragona: Universitat Rovira i Virgili.

Cantero, F. J. (2002). Teoría y análisis de la entonación. Barcelona: Ediciones de la Universidad de Barcelona.

Dorta, J. (ed.) (2013). Estudio comparativo preliminar de la entonación de Canarias, Cuba y Venezuela. Santa Cruz de Tenerife: La Página ediciones.

Escandell-Vidal, Ma. V. (2009). La interrogación en español: semántica y pragmática (Tesis doctoral). Universidad Complutense de Madrid. Disponible en: http://eprints.ucm. es/8139/. Acceso: 09.03.2015.

Fletcher, J., Grabe, E., y Warren, P. (2006). Intonational variation in four dialects of English: the high rising tune. En J. Sun-Ah (ed.), Prosodic typology: the phonology of intonation and phrasing (p. 390-409). Oxford: Oxford University Press.

Font-Rotchés, D. (2007). L'entonació del català. Barcelona: Publicacions de l'Abadia de Montserrat. 
Frota, S., y Prieto, P. (eds.) (2015). Intonation in Romance. Oxford: Oxford University Press.

Grabe, E. (1998). Comparative intonational phonology: English and German (Tesis doctoral). Katholieke Universiteit Nijmegen, Países Bajos. Disponible en: http:// www.phon.ox.ac.uk/files/people/grabe/thesis.html. Acceso: 09.03.2015.

Grabe, E., Kochanski, G., y Coleman, J. (2005). The intonation of native accent varieties in the British Isles - potencial for miscommunication? En K. Dziubalska-Kołaczyk y J. Przedlacka (eds.), English pronunciation models: A changing scene (p. 311-337). Berna: Peter Lang.

Grice, M., Reyelt, M., Benzmüller, R., Mayer, J., y Batliner, A. (1996). Consistency in transcription and labelling of German intonation with GtoBI. En T. Bunnell y W. J. Idsardi (eds.), Proceedings of the 4th International Conference on Spoken Language Processing (vol. 4, p. 1716-1719). Filadelfia: Penn.

Grice, M., Baumann, S., y Benzmüller, R. (2005). German intonation in autosegmentalmetrical phonology. En J. Sun-Ah (ed.), Prosodic typology: The phonology of intonation and phrasing (p. 55-83). Oxford: Oxford University Press.

Gussenhoven, C. (1988). Adequacy in intonation analysis: The case of Dutch. En H. van der Hulst y N. Smith (eds.), Autosegmental studies on pitch accent (p. 95-121). Dordrecht: Foris Publications Holland.

Gussenhoven, C. (2005). Transcription of Dutch intonation. En J. Sun-Ah (ed.), Prosodic typology: The phonology of intonation and phrasing (p. 118-145). Oxford: Oxford University Press.

Kügler, F. (2004). Dialectal variation in question intonation: the case of Swabian and Upper Saxon German. En B. Gunnarsson, L. Bergström, G. Eklund, S. Fridell, L. H. Hansen, A. Karstadt, B. Nordberg, E. Sundgrenand y M. Thelander (eds.), Language variation in Europe. Papers from the Second International Conference on Language Variation in Europe (ICLaVE) (vol. 2, p. 227-240). Uppsala: Uppsala University Press. Disponible en: http://www.ling.uni-potsdam.de/-kuegler/docs/ iclave2_kuegler_fin2.pdf. Acceso: 17.06.2014.

Kügler, F. (2007). The intonational phonology of Swabian and Upper Saxon. Tübingen: Max Niemeyer Verlag.

Ladd, D. R. (1996). Intonational phonology. Cambridge: Cambridge University Press. Leeman, A. (2012). Swiss German intonation patterns. Ámsterdam: John Benjamins.

Martínez Celdrán, E., y Fernández Planas, A. M. (eds.) (2003-2012). AMPER-CAT. Atles multimèdia de la prosòdia de l'Espai Romànic. Disponible en: http://stel.ub.edu/ labfon/amper/index_ampercat_cat.html. Acceso: 01.12.2015.

Mayer, J. (1995). Transcription of German intonation: The Stuttgart System. Informe técnico. Institut für Maschinelle Sprachverarbeitung, Universität Stuttgart. Disponible en: http://www.ims.uni-stuttgart.de/institut/arbeitsgruppen/phonetik/ joerg/labman/STGTsystem.html. Acceso: 01.10.2014. 
Peters, J. (2005). Intonation. En M. Wermke, K. Kunzel-Razum y W. ScholzeStubenrecht (eds.), Duden. Die Grammatik (p.95-128). Mannheim: Dudenverlag. Peters, J. (2006). Intonation deutscher Regionalsprachen. Berlín: de Gruyter.

Petrone, C., y Niebuhr, O. (2014). On the intonation of German intonation questions. The role of the prenuclear region. Language and Speech, 57(1), 108-146. Disponible en: http://las.sagepub.com/content/57/1/108. Acceso: 09.07.2014.

Pierrehumbert, J. B. (1980). The phonology and phonetics of English intonation (Tesis doctoral). Cambridge, Massachusetts: MIT Press. Disponible en: http://hdl.handle. net/1721.1/16065. Acceso: 12.06.2014.

Ramírez Verdugo, Ma a D., Astruc, L., y Morán, Maa B. (2007). Entonación interrogativa en español y en inglés. En J. Dorta (ed.), La prosodia en el ámbito lingüistico románico (p. 95-128). Santa Cruz de Tenerife: La Página Ediciones.

Rossano, F. (2010). Questioning and responding in Italian. Journal of Pragmatics, 42, 2756-2771.

Uhmann, S. (1991). Fokusphonologie: eine Analyse deutscher Intonationskonturen im Rahmen der nicht-linearen Phonologie. Tübingen: Niemeyer. 